\title{
Notizia dell'ultima ora: disponibile anche in Italia l'unica terapia specifica per il Rene Policistico Autosomico Dominante (ADPKD)
}

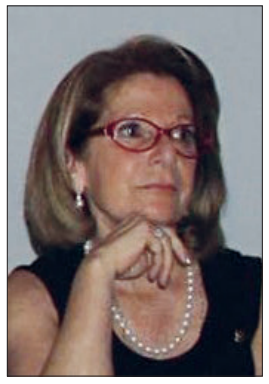

Luisa Sternfeld Pavia

\section{Cari Amici,}

Con molta emozione e poco prima che venga chiuso il giornale, sono finalmente ad annunciare una buona notizia per i pazienti affetti da rene policistico autosomico dominante (ADPKD). Con la pubblicazione sulla Gazzetta Ufficiale, avvenuta oggi, 11 settembre, è infatti disponibile anche nel nostro Paese, tolvaptan (Jinarc@), I'unica terapia specifica per questa patologia, capace di rallentarne la progressione. Si tratta di un traguardo importante, che arriva alla fine di un percorso lungo, caratterizzato da ostacoli e da fasi di stallo, durante il quale l'Associazione, i pazienti e i loro familiari non hanno mai smesso di lottare e di far sentire la propria voce.

Scoperto e sviluppato da Otsuka Pharmaceutical, tolvaptan è stato approvato per la prima volta nel 2014 in Giappone; quindi in Canada e nel 2015 ha ricevuto la raccomandazione del Comitato per i Medicinali per uso umano (CHMP) dell'Agenzia europea per i medicinali (EMA).

Negli anni successivi, il farmaco veniva via via reso disponibile in numerosi Paesi europei, tra cui la Germania, l'Inghilterra, la Scozia, il Lussemburgo, la Francia, il Belgio, la Svizzera, i Paesi Bassi e la Spagna.

Nel frattempo nel nostro Paese continuavamo ad aspettare la rimborsabilità della terapia e a mantenere alta l'attenzione sulla malattia con numerose attività durante tutte le fasi del percorso approvativo durato circa due anni. Abbiamo dato vita, come ricorderete, a \#STOPalrenepolicistico, una petizione volta a fare luce sulla gravità della malattia, sul suo peso emotivo e psicologico e a richiedere l'accesso immediato a

Accepted: September 8, 2017

Published online: September 28, 2017

Indirizzo per la corrispondenza:

Dr.ssa Luisa Sternfeld Pavia

AIRP Associazione Italiana Rene Policistico onlus

Via Antonio Bazzini, 2

20131 Milano

luisa.sternfeld.airp@renepolicistico.it

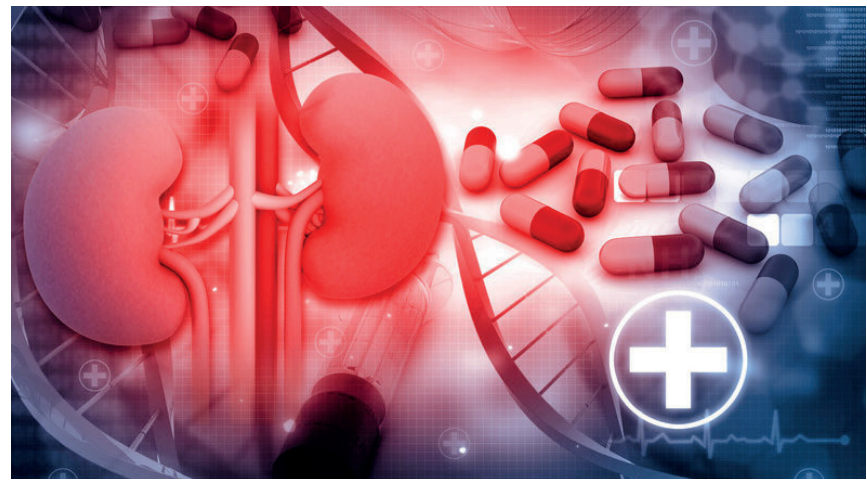

cure innovative. L'iniziativa che ha raccolto oltre cinque mila firme ha ricevuto anche il supporto della comunità scientifica, venendo sottoscritta, tra gli altri, da 110 autorevoli nefrologi italiani. E ancora, abbiamo portato in Italia la campagna internazionale \#BumpPKD per richiamare l'attenzione dell'opinione pubblica sulla malattia. A inizio 2017 abbiamo festeggiato un altro grande risultato. II rene policistico autosomico dominante è stato infatti inserito all'interno dei LEA (Livelli essenziali di assistenza) come malattia cronica e invalidante, permettendo così ai pazienti di avere il diritto all'esenzione di alcune delle principali prestazioni. Un risultato importante, al quale si aggiunge quello appena annunciato: la rimborsabilità di tolvaptan rappresenta infatti un grande passo in avanti nel trattamento del rene policistico autosomico dominante.

Se prima le uniche prospettive per le persone affette da rene policistico erano la dialisi o il trapianto di rene ora, con tolvaptan i pazienti che all'inizio del trattamento si trovano in uno stadio di patologia CKD 2-3a, che sono in un'età compresa tra i 18 e i 50 anni e che hanno un'evidenza di malattia in rapida progressione, possono contare su una terapia in grado di rallentare la progressione della malattia. Tolvaptan (Jinarc(C), ha infatti dimostrato di essere capace di ridurre ogni anno del $32 \%$ il declino della funzionalità renale, di far diminuire del 49\% l'aumento annuo del volume renale totale nell'arco di tre anni ${ }^{1}$ e di essere efficace anche sul controllo del dolore.

${ }^{1}$ Torres VE, Chapman AB Devuyst O, et al; TEMPO 3:4 Trial Investigators. Tolvaptan in patients with autosomal dominant polycystic kidney disease. N Engl J Med. 2012;367 (25): 2407-18. 
Ma quali sono i passi da fare per poter accedere al farmaco? Nei prossimi mesi, le varie Regioni e ASL decideranno le modalità e le tempistiche di disponibilità di tolvaptan (Jinarc(C), in linea con il tradizionale iter che segue la pubblicazione di un farmaco sulla Gazzetta Ufficiale. Contemporaneamente e indipendentemente dalla disponibilità del farmaco nelle varie Regioni, i medici qualora lo ritenessero opportuno potranno effettuare una richiesta nominale per i pazienti elegibili al trattamento. Le richieste dei medici saranno poi valutate caso per caso.

Purtroppo il rene policistico è una malattia poco conosciuta, molto spesso sottovalutata, che in Italia viene diagnosticata in uno stadio avanzato, tra i 30 e i 55 anni, quando la funzionalità del rene può essere già compromessa. Sappiamo inoltre come la malattia possa avere conseguenze pesanti anche sul resto dell'organismo: al rene policistico autosomico dominante si possono infatti accompagnare complicanze renali, come ipertensione in età precoce e dolore, comparsa di cisti anche nel fegato e nel pancreas e una possibile maggiore insorgenza di aneurismi cerebrali. Non possiamo non citare anche l'impatto sociale e psicologico della malattia. A causa dell'ingrossamento dei reni, spesso si convive con dolore cronico, depressione e difficoltà nello svolgere le attività quotidiane. Una recente indagine ha infatti messo in luce come un paziente su tre veda compromesse le proprie relazioni sociali (41\%), la vita sessuale $(42 \%)$ e rinunci ad avere figli $(35 \%)^{2}$. II rene policistico ha un impatto pesante sia a livello fisico sia a livello sociale, ma che ora, grazie all'arrivo di Jinarc (C), i pazienti italiani elegibili al trattamento, possono finalmente affrontare.

Ma la nostra lotta al rene policistico non si ferma qui, perché la speranza non deve mai spegnersi. Prenderanno presto il via nuovi trial clinici per la ricerca e lo sviluppo di altri trattamenti, perché tutti coloro che sono affetti dalla malattia devono poter avere di fronte a sé una sola direzione: si va avanti!

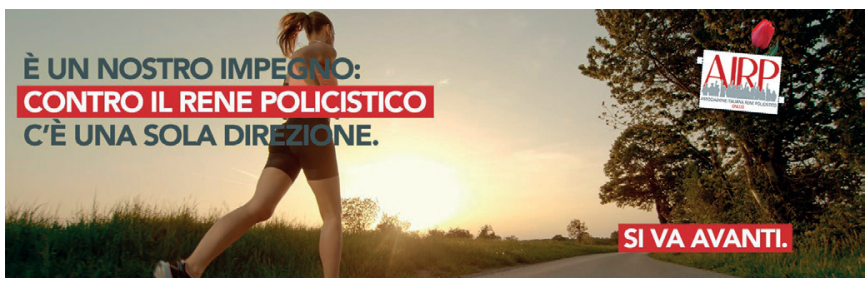

Luisa Sternfeld Pavia Presidente AIRP 